\title{
QTL mapping for Chinese northern-style steamed bread specific volume
}

\author{
Peng Wu ${ }^{{ }^{1}, 2}$, Bin Liu ${ }^{* 1}$, Tao Zhou ${ }^{* 3}$, Zhuokun $\mathrm{Li}^{1},{\text { Haiyun } \mathrm{Du}^{4}, \text { Jiteng Wang }}^{5}$, Jichun Tian ${ }^{1,6}$ \\ ${ }^{1}$ Group of Quality Wheat Breeding of State Key Laboratory of Crop Biology of Shandong Agricultural University, Tai’an, China; \\ ${ }^{2}$ College of Food Science and Engineering, Shandong Agricultural University, Tai’an, China; \\ Corresponding Author: wupengguai@163.com \\ ${ }^{3}$ College of Horticulture Science and Engineering, Shandong Agricultural University, Tai'an, China; \\ ${ }^{4}$ Tai'an Bureau of Quality and Technical Supervision, Tai'an, China; \\ ${ }^{5}$ Rongcheng Entry-Exit Inspection and Quarantine Bureau, Rongcheng, China; \\ ${ }^{6}$ Agronomy Department, Shandong Agricultural University, Tai’an, China; \\ Corresponding Author: jctian@sdau.edu.cn, jctian9666@126.com
}

Received 25 April 2011; revised 26 June 2011; accepted 7 July 2011.

\begin{abstract}
In this study, quantitative trait loci (QTLs) with additive effects, epistatic effects for CNSB specific volume in bread wheat (Triticum aestivum L.), were studied in cultivars Huapei 3 and Yumai 57 (Triticum aestivum L.). The DH population and the parents were planted in 2007 and 2008 in Tai'an and 2008 in Suzhou. QTL analyses were performed using the software of IciMapping v2.2 based on the mixed linear model. Five putative QTLs for CNSB specific volume were detected on 5 chromosomes where single QTLs explained $5.11 \%$ to $9.75 \%$ of phenotypic variations. All of them had negative effects on specific volume and were contributed by Yumai 57 alleles. Qsv-1B was detected in both environment 1 and 3 with $13.88 \%$ and $4.83 \%$ phenotypic variations which had positive effects and was transmitted by Huapei 3 alleles. Fourteen pairs of QTLs with epistatic effects were detected for specific volume. Seven major QTLs, Qsv-1B/Qsv-3A, Qsv-2D/Qsv-3A, Qsv-3A/Qsv5B1, Qsv-1B/Qsv-6D, Qsv-2D/Qsv-4D, Qsv-4A/ Qsv-6B and Qsv-3A/Qsv-7D could account for $13.88 \%, 20.39 \%, 18.88 \%, 12.31 \%, 18.78 \%, 11.98 \%$, and $17.05 \%$ of the phenotypic variation of specific volume. The information obtained in this study will be useful for manipulating the QTLS for CNSB specific volume property by molecular marker-assisted selection (MAS).
\end{abstract}

Keywords: Wheat; Doubled Haploid; Quantitative Trait Loci; CNSB; Specific Volume

"Peng Wu and Bin Liu, Tao Zhou contributed equally to this work.

\section{INTRODUCTION}

Chinese steamed bread (CSB) is a staple food of the Chinese people, especially in northern China, where it is eaten at every meal. It has been consumed for at least 2,000 years in China, and has also been gaining in popularity in Korea, Japan, and some Southeast Asian countries in recent years as food culture intercommunications have rapidly developed among different countries. There are two types of steamed bread in China: northern-style (CNSB) and southern-style (CSSB), with differences in the ingredients and quality evaluation. Most research has focused on CNSB, since it is by far the most widely produced, and about $40 \%$ of Chinese wheat consumption is used to make CNSB, particularly in northern China [1].

CNSB quality is defined by its specific volume, external appearance, internal characteristics, and color. Specific volume is an important factor in CNSB's score, which is the ratio of volume to weight. The greater the value, the better leavened is the CNSB. It has significant correlations with color, another important factor in CNSB's scores [2]. However, few QTL analyses have yet been reported for those steamed bread's specific volume.

Huapei 3 is hard wheat with a higher grain protein content and wet gluten content. Yumai 57 is soft wheat with higher gluten index and sodium dodecyl sulfate (SDS) sedimentation volume and is more productive under different ecological conditions [3]. The parents both have white seed coats. Wheat processing and enduse characteristics, collectively known as quality traits, are controlled by both genetics and environment. Molecular-genetic studies to elucidate this control may increase the efficiency of breeding wheat for improved quality [4]. Quantitative trait loci (QTLs) analyses of 
quality traits have been reported using a set of 168 doubled haploid (DH) lines derived from a cross of Huapei 3 $\times$ Yumai 57.

The objectives of the study described here was to detect QTLs with additive effects, epistatic effects, and QEs for the specific volume of CNSB, which should be useful in manipulating the QTLs for steamed-bread specific volume by the molecular marker-assisted selection in wheat breeding programs.

\section{MATERIALS AND METHODS}

\subsection{Plant Materials and Growth Conditions}

A population of $168 \mathrm{DH}$ lines, derived from a cross between two Chinese wheat cultivars Huapei 3 (Hp3) and Yumai 57 (Ym57), was used for the construction of a linkage map. The DH population and parents were kindly provided by Professor Yan Hai, Henan Academy of Agricultural Sciences, Zhengzhou, China. Hp3 and Ym57 were released by Henan Province in 2006 [5] and by the State (China) in 2003 [6], respectively. The two parents are cultivated over a large area in the HuangHuai Wheat Region in China, differing in several agronomically important traits as well as in baking quality traits.

The $168 \mathrm{DH}$ lines and parents were planted in two replications at each location with a completely randomized block design at three environments in 2007 and 2008 in Tai' an $\left(36.18^{\circ} \mathrm{N}, 117.13^{\circ} \mathrm{E}\right)$, Shandong Province; in 2007 in Suzhou $\left(31.32^{\circ} \mathrm{N}, 120.62^{\circ} \mathrm{E}\right)$, Anhui Province. In Tai'an, there were remarkable differences in temperature and soil conditions between the years 2007 and 2008.

In autumn 2007, all lines and parental lines were grown in $2 \mathrm{~m}$ long by three-row plots (25 cm apart); in autumn 2008, the lines were grown in $2 \mathrm{~m}$ long by four-row plots ( $25 \mathrm{~cm}$ apart). The soil was brown earth, in which the available $\mathrm{N}, \mathrm{P}$, and $\mathrm{K}$ contents in the top 20 $\mathrm{cm}$ were $40.2 \mathrm{mg} / \mathrm{kg}, 51.3 \mathrm{mg} / \mathrm{kg}$ and $70.8 \mathrm{mg} / \mathrm{kg}$. Before planting, $27,500 \mathrm{~kg} /$ hectare (ha) of farmyard manure or barnyard manure (nitrogen content, $0.05 \%$ $0.1 \%$ ), $225 \mathrm{~kg} / \mathrm{ha}$ of urea, $300 \mathrm{~kg} / \mathrm{ha}$ of phosphorus diamine fertilizer, $225 \mathrm{~kg} / \mathrm{ha}$ of potassium chloride, and 15 $\mathrm{kg} / \mathrm{ha}$ of zinc sulphate were added as fertilizers. In Tai'An, the rainfalls during the growth cycles were 165 $\mathrm{mm}$ and $172.5 \mathrm{~mm}$ in 2007-2008 and 2008-2009, respectively. In Suzhou, the rainfalls during the growth cycle totaled $207.5 \mathrm{~mm}$ in 2008-2009.

All of crop management followed local practices. Plots were irrigated in winter (December 1, 2007), and at jointing (April 3, 2008), anthesis (May 7, 2008), and grain filling (May 20, 2008). They were top-dressed with $225 \mathrm{~kg} /$ hectare and $75 \mathrm{~kg} /$ hectare urea at the jointing (April 3, 2008) and anthesis stages (May 7, 2008) with irrigation, respectively. The lines were harvested individually at maturity to prevent yield loss from over-rip- ening. Harvested grain samples from the two replicates at each environment were mixed and cleaned prior to conditioning. One thousand grams of grain samples from each line at each environment were milled on a Buhler experimental mill (model-MLU 300 m/s Buhler, Uzwil Switzerland). Prior to milling, the hard, medium hard (mixtures of hard and soft wheat) and soft wheats were tempered to around $16 \%, 15 \%$, and $14 \%$ moisture contents for 24 hours, respectively.

\subsection{Steamed Bread Preparation}

CNSB was prepared according to the Chinese standard SB/T10139-93 Appendix A [7]. The milled sample, $100 \mathrm{~g}$ of flour, was mixed with the yeast solution (1g dry yeast dispersed in $48 \mathrm{ml} 38^{\circ} \mathrm{C}$ water). Dough was fermented for $60 \mathrm{~min}$ in a fermentation cabinet $\left(38^{\circ} \mathrm{C}, 85 \%\right.$ rh) after 3 min mixing. The fermented dough was divided into two pieces and each shaped by hand for 3 min into a round dough piece with a smooth surface. Then the breads were steamed for $20 \mathrm{~min}$ in a steam chamber $\left(>100^{\circ} \mathrm{C}\right.$ ) after resting in the air for $15 \mathrm{~min}$. Then the steamed breads were cooled in the air for $40 \mathrm{~min}$.

\subsection{Specific Volume Measurements of CNSB Samples}

Steamed bread was placed in a covered bamboo container at room temperature immediately after steaming. Then the steamed breads were cooled in the air for 40 min. Samples were measured using an electric balance for weight and a substitution method with rapeseed for volume. All scores were taken three times per sample. The mean values of CNSB specific volume in each environment were used for statistical analyses.

All determinations were made at least three times, and were expressed on a $14 \%$ - $16 \%$ moisture basis.

\subsection{DNA Marker Assay and Construction of the Genetic Map}

A genetic linkage map of $\mathrm{DH}$ population with 323 markers, including 284 SSR loci, 37 EST loci, 1 intersimple sequence repeat (ISSR) locus, and 1 HMW-GS locus was constructed. This linkage map covered a total length of 2,485.7 $\mathrm{cm}$ with an average distance of 7.67 $\mathrm{cm}$ between adjacent markers. Thirteen markers remained unlinked. These linked markers formed 24 linkage groups at LOD 4.0. The genomic locations of marker loci/linkage groups were determined based on the wheat consensus map. Thirty loci (7.03\%) were mapped on different chromosomal locations compared with the map published by Somers et al. [8]. The recommended map distance for genome wide QTL scanning was an interval length less than $10 \mathrm{~cm}$ [9]. In this 
study, the interval length was $7.67 \mathrm{~cm}$, so the map was deemed suitable for genome-wide QTL scanning.

The genetic map was constructed with MAPMAKER/ Exp version 3.0b [10]. First, the 'group' command was used to divide the markers in the sequence into linkage groups at a LOD of 4.0. Then, the 'compare' command was used to compute the maximum likelihood map for each specified order of markers, and to report the orders sorted by the likelihoods of their maps. The 'try' and 'ripple' commands were used to add markers to framework maps and to check the final marker order. Unlinked groups were oriented and placed for the same chromosome based on the microsatellite consensus map [8]. The Kosambi mapping function [11] was used to convert recombination fractions into $\mathrm{cm}$ as map distances. The linkage map was finally drawn using the software Mapchart, version 2.1 [12].

\subsection{Statistical Analysis}

Analysis of variance (ANOVA) was carried out using the SPSS version 13.0 (SPSS, Chicago, USA) program. QTLs with additive effects and epistatic effects as well as QEs in the DH population were mapped by the software QTLNetwork, version 2.0 [13], based on the mixed linear model [14]. Composite interval analysis was undertaken using forward-backward stepwise, multiple linear regression with a probability into and out of the model of 0.05 and window size set at $10 \mathrm{~cm}$. Significant thresholds for QTL detection were calculated for each data set using 1000 permutations and a genome-wide error rate of 0.10 (suggestive) and 0.05 (significant). The final genetic model incorporated significant additive effects and epistatic effects as well as their environmental interactions.

Each QTL was named for steamed bread's specific volume by the first two letters with the relevant chromosomal number such as "Qsv". If there were more than one QTL on a chromosome, the serial number was added after the chromosomal number, separated by a hyphen. The positions of these QTLs were indicated by the marker interval bracketing the concerned QTL with the estimated distance $(\mathrm{cm})$ from the left marker.

\section{RESULTS AND DISCUSSION}

\subsection{Statistical Analysis of the Phenotypic Assessments}

The DH population had a wide range and considerable variation for CNSB specific volume traits. Mean values for CNSB specific volume traits for the $\mathrm{DH}$ population and the parents in three environments are shown in Table 1. The mean of CNSB specific volume was between that of Hp3 and Ym57, which expressed the existence of the large transgressive segregation, whereas the phenoltypic variations among the $\mathrm{DH}$ lines were obvious. Transgressive segregants were observed in all the environments, with some lines higher or lower than the parents. The specific volume traits among the $\mathrm{DH}$ population segregated continuously, and followed a normal distribution (Figure 1), and both absolute values of skewness and kurtosis were less than 1.0 (Table 1), indicating its polygenic inheritance and suitability of the data for QTL analysis [15].

Mean, SE, Max, Min, SD, Skew, and Kurt are the average, standard error, maximum, minimum, standard deviation, skewness, and kurtosis of all observations for the $\mathrm{DH}$ population in the three environments.

\subsection{QTL Analyses}

Five additive QTLs and fourteen pairs of epistatic effects were identified for specific volume of CNSB in three years (Tables 2 and 3) by using the software of QTLNet-work version 2.0 [13] and IciMapping v2.2 [16], based on the mixed linear model.

Seven QTLs (Qsv-1B/Qsv-3A Xbarc119-Xgwm18/ Xbarc321-Xswes107, Qsv-2D/Qsv-3A Xcfd53-Xwmc18/ Xbarc321-Xswes107, Qsv-3A/Qsv-5B1 Xwmc264Xcfa2193/Xgwm213-Xswes861.2, Qsv-1B/Qsv-6D Xbarc061-Xwmc766/Xswes679.1-Xcfa2129, Qsv-2D/

Table 1. Phenotypic performance of wheat CNSB specific volume in three environments.

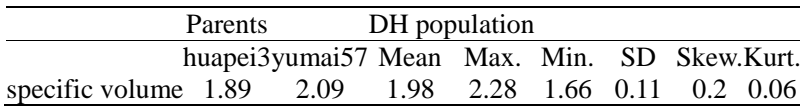

Table 2. Estimated additive (A) of QTLs for steamed bread specific volume in the three environments.

\begin{tabular}{cccccccc}
\hline Trait & Chrom & Site (cM) & LeftMarker & RightMarker & Additive effects & LOD & Contributions (\%) \\
\hline specific volume $^{1}$ & Qsv-6A & 68 & Xwmc553 & Xgwm732 & -0.06 & 2.31 \\
specific volume $^{2}$ & Qsv-7B2 & 13 & Xwmc273.1 & Xcfd22.1 & -0.06 & 3.12 \\
specific volume $^{2}$ & Qsv-7D & 75 & Xbarc352 & Xgwm295 & -0.05 & 2.461 \\
specific volume $^{3}$ & Qsv-1A & 54 & Xcfd59 & Xwmc402.2 & -0.04 & 2.191 \\
specific volume $^{3}$ & Qsv-3D & 94 & Xbarc071 & Xgwm114 & -0.06 & 5.11 & 3.711 \\
\hline
\end{tabular}

${ }^{1} 2007$ Tai'An, ${ }^{2} 2008$ Tai'An, ${ }^{3} 2007$ Suzhou. 


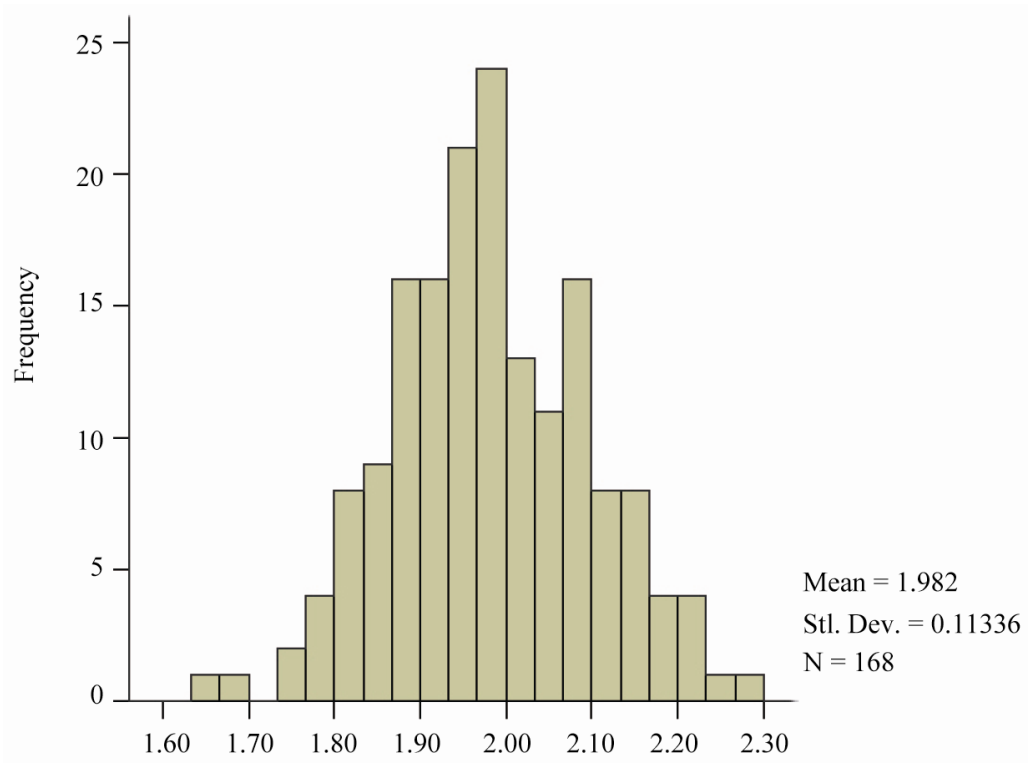

Figure 1. Frequency distribution of steamed bread specific volume related traits in 168 doubled haploid lines derived from the cross of Huapei $3 \times$ Yumai 57 evaluated at 3 environments.

Table 3. Estimated epistasis (AA) of QTLs for steamed bread specific volume in the three environments.

\begin{tabular}{|c|c|c|c|c|c|c|c|c|c|}
\hline Trait & $\frac{\text { QTL }}{1}$ & $\frac{\text { Site }(\mathrm{cm})}{1(\mathrm{~cm})}$ & Flanking marker & $\frac{\mathrm{QTL}}{2}$ & $\frac{\text { Site }(\mathrm{cm})}{2(\mathrm{~cm})}$ & Flanking marker & Epistatic effects & LOD & $\begin{array}{c}\text { Contributions } \\
(\%)\end{array}$ \\
\hline specific volume $^{1}$ & Qsv-1B & 34 & Xbarc119-Xgwm18 & Qsv-3A & 26 & Xbarc321-Xswes107 & 0.07 & 3.13 & 13.88 \\
\hline specific volume $^{1}$ & Qsv-2D & 26 & Xcfd53-Xwmc18 & Qsv-3A & 16 & Xbarc321-Xswes107 & 0.08 & 4.08 & 20.39 \\
\hline specific volume $^{1}$ & Qsv-6D & 0 & Xwmc412.1-Xcfd49 & Qsv-7A & 86 & Xwmc530-Xcfa2123 & 0.05 & 3.39 & 8.58 \\
\hline specific volume $^{2}$ & Qsv-1A & 48 & Xwmc550-Xbarc269 & Qsv-3A & 174 & Xcfa2170-Xbarc51 & 0.06 & 3.41 & 7.83 \\
\hline specific volume $^{2}$ & Qsv-1B & 76 & Xbarc061-Xwmc766 & Qsv-6D & 144 & Xswes679.1-Xcfa2129 & 0.08 & 3.14 & 12.31 \\
\hline specific volume $^{2}$ & Qsv-2D & 130 & Xbarc129.2-Xcfd50 & Qsv-4D & 28 & Xwmc331-Xgwm194 & 0.1 & 3.15 & 18.78 \\
\hline specific volume $^{2}$ & Qsv-4A & 2 & Xwmc718-Xwmc262 & Qsv-6B & 26 & Xbarc247-Xbarc1129 & -0.09 & 3.03 & 11.98 \\
\hline specific volume $^{2}$ & Qsv-6A & 84 & Xcfe179.2-Xcfe179.1 & Qsv-6B & 8 & Xcfa2187-Xgwm219 & -0.07 & 3.84 & 8.54 \\
\hline specific volume $^{3}$ & Qsv-1A & 86 & Xgwm498-Xcwem6.2 & Qsv-5A & 30 & Xswes45-Xbarc180 & -0.05 & 7.48 & 7.37 \\
\hline specific volume $^{3}$ & Qsv-1B & 38 & Xcwem9-Xbarc120.3 & Qsv-6D & 108 & Xgwm133.2-Xswes861.1 & 0.07 & 3.42 & 4.83 \\
\hline specific volume $^{3}$ & Qsv-3A & 0 & Xbarc310-Xbarc321 & Qsv-7D & 78 & Xbarc352-Xgwm295 & -0.08 & 15.89 & 17.05 \\
\hline specific volume $^{3}$ & Qsv-3D & 0 & Xcfd34-Xbarc376 & Qsv-7A & 90 & Xwmc530-Xcfa2123 & -0.04 & 5.18 & 5.14 \\
\hline specific volume $^{3}$ & Qsv-5B2 & 2 & Xbarc36-Xbarc140 & Qsv-7A & 22 & Xgwm60-Xbarc070 & -0.05 & 7.56 & 9.39 \\
\hline
\end{tabular}

${ }^{1} 2007$ Tai'An, ${ }^{2} 2008$ Tai'An, ${ }^{3} 2007$ Suzhou

Qsv-4D Xbarc129.2-Xcfd50/Xwmc331-Xgwm, Qsv-4A/ Qsv-6B Xwmc718-Xwmc262/Xbarc247-Xbarc1129 and Qsv-3A/Qsv-7D Xbarc310-Xbarc321/Xbarc352-Xgwm295) explained the phenotypic variance respectively for 13.88\%, 20.39\%, 18.88\%, 12.31\%, 18.78\%, 11.98\%, and 17.05\% (Table 3). All of them were major QTLs and could be used in the molecular marker-assisted selection (MAS) in wheat breeding programs.

The QTL Qsv-1B was detected both in environment-1 and environment-3, which explained the phenotypic variance of $13.88 \%$ and $4.83 \%$.

Five additive QTLs were detected for specific volume on five chromosomes 6A, 7B2, 7D, 1A and 3D (Figure 2). They explained phenotypic variance from 5 . $11 \%$ to 9.75\%. All of the five QTLs had negative effects on spe- cific volume and were contributed by Yumai 57 alleles that have the higher specific volume. The total additive QTL detected for specific volume accounted for $38.454 \%$ of the phenotypic variance.

Fourteen pairs of epistatic effects were identified for specific volume and located on chromosomes 1B-3A, 2D-3A, 3A-5B1, 6D-7A, 1A-3A, 1B-6D, 2D-4D, 4A-6B, 6A-6B, 1A-5A, 1B-6D, 3A-7D, 3D-7A and 5B2-7A (Figure 3). They explained the phenotypic variance ranging from $4.83 \%$ to $20.39 \%$. Qsa1B/ Qsa3D-2 and Qsa2A/Qsa3A had negative effects of -0.1525 and -0.1516 . The general contribution of three pairs of epistatic QTLs was 164.95\%.

QTLs for the specific volume of wheat products have been reported briefly by some authors. The study of 

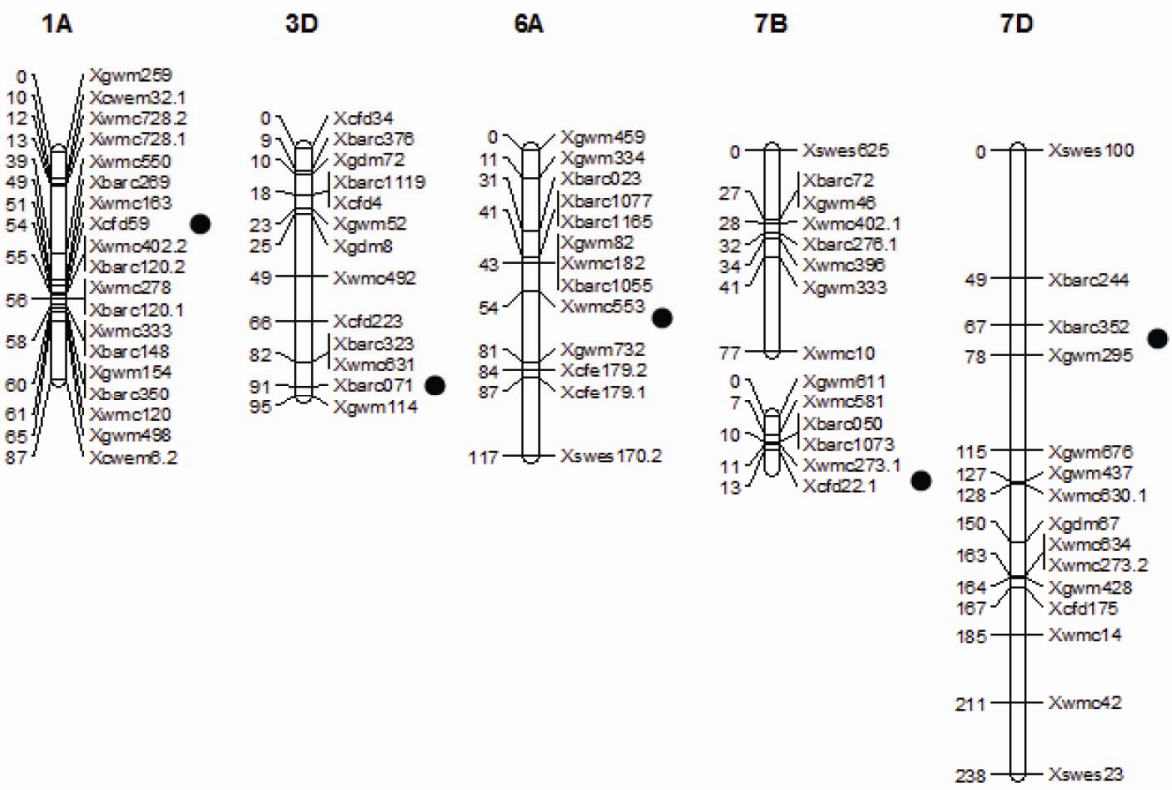

Figure 2. Positions of additive QTLs conferring steamed bread specific volume in 168 doubled haploid lines derived from the cross of Huapei $3 \times$ Yumai 57 evaluated in the three environments.

QTLs for the volume of bread of the RIL population derived from two India wheat cultivars "HI 977" and "HD 2329" as parents, shows that the most influenced QTLs are located on chromosomes 6B and 6D, while other QTLs are located on chromosomes 1B, 1D, 2A, 3A, $5 \mathrm{~B}$, and 5D [17]. This was similar to our conclusions.

As far as the specific volume of steamed bread in China, only Fan's study has been reported, which detected the QTLs for the specific volume on 6B Xgwm193-Xgwm608b [18]. That was not consistent with our results. A number of factors contributed to the difference as follows, different population and experimental conditions, different QTL analyses methods, and no allele variation for corresponding traits in parents.

The five QTLs with additive effects for the specific volume of CNSB had negative effects and were contributed by Yumai 57 alleles in our study.

No additive effects were detected in most of the QTLs (86\%) for the specific volume of CNSB with epistatic effects. Two QTLs (Qsv-1A-Qsv-5A with 7.37\% contributions and Qsv-3A-Qsv-7D with $17.05 \%$ contriburions) were involved in additive effects in the detected 14 pairs of epistatic QTLs. This indicates that several loci involved in epistatic interactions may not have significant effects for CNSB specific volume alone, and may affect the trait expression by epistatic interactions. These results suggest that some of the additive QTLs may be detected with effects confounded by epistatic effects, if the epistatic effects were ignored in QTL mapping.

Qsv-1B was detected for CNSB specific volume in our study in environment- 1 and environment-3 accounting for $13.88 \%$ and $4.83 \%$ of the phenotypic variations which had positive effects and were contributed by Huapei 3 alleles. Thus, breeders must take into account such complexity and examine the effects of individual loci in the targeted genetic background, in order to obtain the expected phenotypes of the genes of interest [19]. The present investigation might be the first report of epistatic QTLs associated with CNSB specific volume using molecular markers. The results indicate that epistasis was an important genetic basis for CNSB specific volume variations.

\section{CONCLUSIONS}

In summary, a total of five putative QTLs and fourteen pairs of QTLs with epistatic effects were detected for specific volume in $168 \mathrm{DH}$ lines derived from a cross Huapei $3 \times$ Yumai 57. Five putative QTLs for CNSB specific volume were detected on 5 chromosomes, where single QTLs explained $5.11 \%$ to $9.75 \%$ of phenotypic variations. All of them had negative effects on specific volume and were contributed by Yumai 57 alleles. Qsv-1B was detected in both environment 1 and 3 with $13.88 \%$ and $4.83 \%$ phenotypic variations, which had positive effects and was transmitted by Huapei 3 alleles. Fourteen pairs of QTLs with epistatic effects were detected for specific volume. Seven major QTLs, Qsv-1B/ Qsv-3A, Qsv-2D/Qsv-3A, Qsv-3A/Qsv-5B1, Qsv-1B/ Qsv-6D, Qsv-2D/Qsv-4D, Qsv-4A/Qsv-6B and Qsv-3A/ Qsv-7D could account for $13.88 \%, 20.39 \%, 18.88 \%$, 


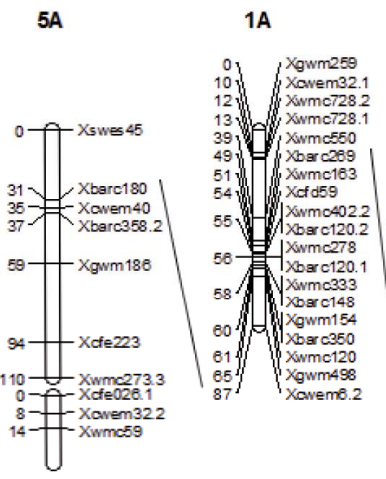

4D

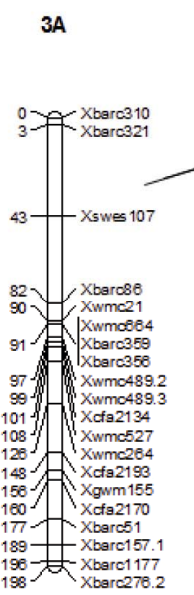

2D
$1 \mathrm{~B}$

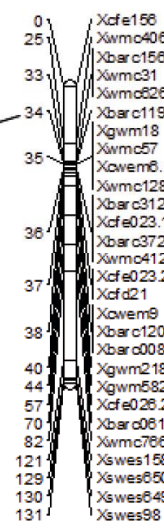

$3 \mathrm{~A}$
$6 \mathrm{D}$

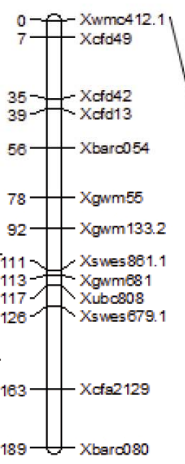

$5 B$
7A

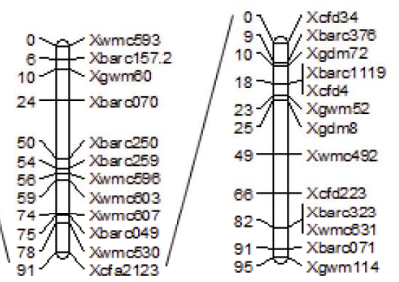

7A

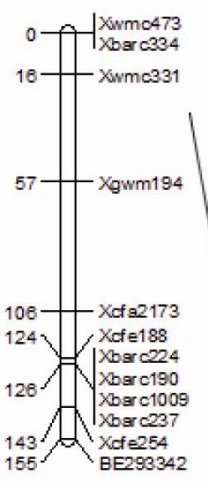

4A

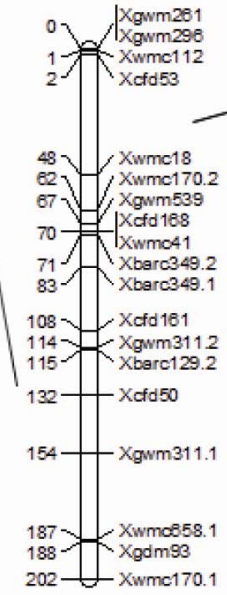

$6 B$

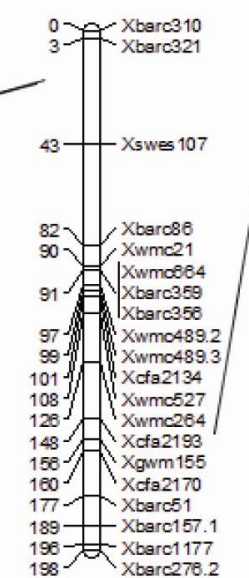

$6 A$
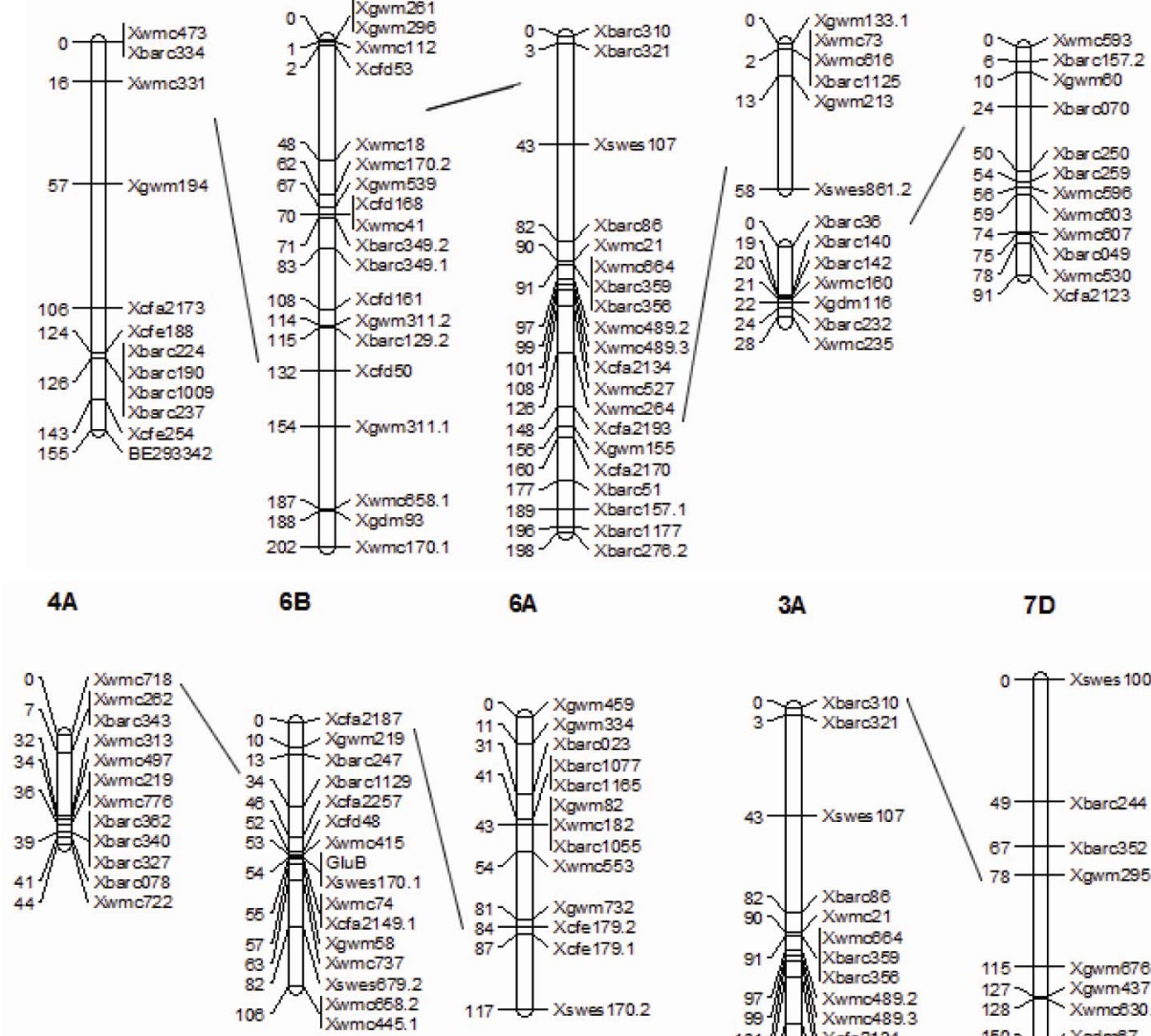

3A

7D

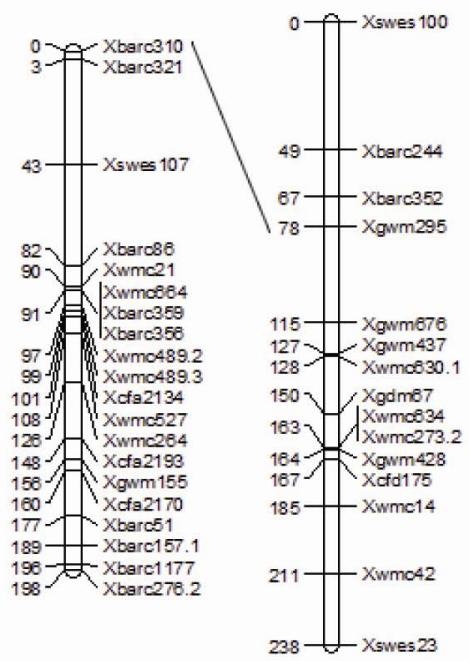

Figure 3. Positions of estimated epistasis QTLs conferring steamed bread specific volume in 168 doubled haploid lines derived from the cross of Huapei $3 \times$ Yumai 57 evaluated in the three environments. 
$12.31 \%, 18.78 \%, 11.98 \%$ and $17.05 \%$ of the phenotypic variation of specific volume. The information obtained in this study will be useful for manipulating the QTLS for CNSB specific volume property by molecular marker-assisted selection (MAS).

\section{ACKNOWLEDGEMENTS}

The authors thank gratefully Dr. C.E. (Chuck) Walker (Kansas State University) for his editorial suggestions and careful reading of the manuscript. The DH population and parents were kindly provided by Professor Yan Hai, Henan Academy of Agricultural Sciences, Zhengzhou, China. Fin ancial support was provided by the 973 program of the National Nature Science Foundation of China (2009CB118301), and the National Natural Science Foundation of China (No. 30971764) from the Ministry of Science and Technology of the People's Republic of China.

\section{REFERENCES}

[1] He, Z.H., Liu, A.H., Peña, R.J. and Rajaram, S. (2003) Suitability of Chinese wheat cultivars for production of northern style Chinese steamed bread. Euphytica, 131, 155-163. doi:10.1023/A:1023929513167

[2] Guo, B.L., Wei, Y.M., Zhang, G.Q., Yang, S.H., Hu, X.Zh. (2002) Study on the quality judging methods of steamed bread. Journal of Triticeae Crops, 22, 7-10

[3] Hai, Y., and Kang, M.H. (2007) Breeding of a new wheat variety Huapei 3 with high yield and early maturing. Henan Agriculture Science, 5, 36-37.

[4] Nelson, J.C. Andreescu, C., Breseghello, F., Finney, P.L., Gualberto, D.G., Bergman, C.J., Pena, R.J., Perretant, M.R., Leroy, P., Qualset, C.O. and Sorrells, M.E. (2006) Quantitative trait locus analysis of wheat quality traits, Euphytica, 149, 145-159.

doi:10.1007/s10681-005-9062-7

[5] Hai, Y. and Kang, M.H. (2007) Breeding of a new wheat variety Huapei 3 with high yield and early maturing. Henan Agriculture Science, 5, 36-37.

[6] Guo, C.Q., Bai, Z.A., Liao, P.A. and Jin, W.K. (2004) New high quality and yield wheat variety Yumai 57. China Seed Industry, 4, 54.

[7] SB/T10139-93 (1993) wheat flour for steamed bread, Appendix A.

[8] Somers, D.J., Isaac, P. and Edwards, K. (2004) A highdensity microsatellite consensus map for bread wheat (Triticum aestivum L.). Theoretical and Applied Genetics, 109, 1105-1114. doi:10.1007/s00122-004-1740-7

[9] Doerge, R.W. (2002) Multifactorial genetics: Mapping and analysis of quantitative trait loci in experimental populations. Nature Reviews, 3, 43-52.

doi:10.1038/nrg703

[10] Lincoln, S.E., Daly, M.J. and Lander, E.S. (1993) Constructing genetic maps with MAPMAKER/EXP version 3.0: A tutorial and reference manual. Whitehead Institute for Biomedical Research, Cambridge.

[11] Kosambi, D.D. (1944) The estimation of map distances from recombination values. Annals of Human Genetics, 12, 172-175. doi:10.1111/j.1469-1809.1943.tb02321.x

[12] Voorrips, R.E. (2002) MapChart: Software for the graphical presentation of linkage maps and QTL. Journal of Heredity, 93, 77-78. doi:10.1093/jhered/93.1.77

[13] Yang, J. and Zhu, J. (2005) Predicting superior genotypes in multiple environments based on QTL effects. Theoretical and Applied Genetics, 110, 1268-1274. doi:10.1007/s00122-005-1963-2

[14] Wang, D.L., Zhu, J., Li, Z.K. and Paterson, A.H. (1999) Mapping QTLs with epistatic effects and QTL environment interactions by mixed linear model approaches. Theoretical and Applied Genetics, 99, 1255-1264. doi:10.1007/s001220051331

[15] Cao, G., Zhu, J., He, C., Gao, Y., Yan, J. and Wu, P. (2001) Impact of epistasis and QTL environment interaction on the developmental behavior of plant height in rice (Oryza sativa L.). Theoretical and Applied Genetics, 103, 153160. doi:10.1007/s001220100536

[16] Li, H., Li, Z. and Wang, J. (2008) Inclusive composite interval mapping (ICIM) for digenic epistasis of quantitative traits in biparental populations. Theoretical and Applied Genetics, 116, 243-260. doi:10.1007/s00122-007-0663-5

[17] Elangovan, M.R., Rai, B.B., Dholakia, M.D., Lagu, R., Tiwari, R.K., Gupta, V.S. Rao, M.S. and Gupta V.S. (2008) Molecular genetic mapping of quantitative trait loci associated with loaf volume in hexaploid wheat (Triticum aestivum). Journal of Cereal Science, 47, 587598. doi:10.1016/j.jcs.2007.07.003

[18] Fan, Y. D., Sun, H.Y., Zhao, J.L., Ma, Y.M., Li, R.J., Li, S.Sh. (2009) QTL mapping for quality traits of northern-style hand-made Chinese steamed bread. Journal of Cereal Science, 49, 225-229. doi:10.1016/j.jcs.2008.10.004

[19] Zhang K.P., Tian J.C., Zhao L., Wang S.S. (2008) Mapping QTLs with epistatic effects and QTL environment interactions for plant height using a doubled haploid population in cultivated wheat. Journal of Genetics \& Genomics, 35, 119-127. doi:10.1016/S1673-8527(08)60017-X 